\title{
TeleCOVID-19: A Multifaceted Strategy from a Public Brazilian Telehealth Service During the COVID-19 Pandemic
}

\author{
Maria Beatriz Moreira ALKMIM ${ }^{\mathrm{a}}$ Milena Soriano MARCOLINO ${ }^{\mathrm{a}, \mathrm{b}}$ \\ Clara Rodrigues Alves de OLIVEIRA ${ }^{\mathrm{a}, \mathrm{b}}$ Isabela Nascimento BORGES ${ }^{\mathrm{a}, \mathrm{b}}$ \\ Clareci Silva CARDOSO ${ }^{c}$ Gustavo Machado ROCHA ${ }^{c}$ Leonardo Bonisson RIBEIRO ${ }^{a}$ \\ Lidiane Aparecida Pereira de SOUSA ${ }^{\text {a }}$ Mayara Santos MENDES ${ }^{a}$ \\ Maria Cristina da PAIXÃO ${ }^{\mathrm{a}}$ Renato Minelli FIGUEIRA ${ }^{\mathrm{a}}$ \\ and Antonio Luiz P. RIBEIROa,b,1 \\ ${ }^{a}$ Telehealth Center, Hospital das Clínicas \\ ${ }^{\mathrm{b}}$ Department of Internal Medicine, Faculdade de Medicina, Universidade Federal de \\ Minas Gerais, Belo Horizonte, Brazil \\ 'School of Medicine, Federal University of São João del-Rei, Divinópolis, Brazil
}

\begin{abstract}
The COVID-19 pandemic has quickly and radically transformed health systems worldwide. The challenges are imposed by the need for social distance, remote management of less severe cases, and the constant need for updating health care professionals and the population with reliable information. We aim to describe the experiences and developments of a Brazilian telehealth public service during the pandemic. Numerous tools have been developed and made available, to be used in an integrated manner, by both health professionals and the general public. Those included a chatbot for guidance, a teleconsultation platform combined with a telemonitoring system, a teleconsulting service, and a tele-education program. The TNMG services appear to be efficient and robust during the health crisis of COVID19, through different tools and methodologies focused on both professionals and users of the health systems.
\end{abstract}

Keywords. Telemedicine, COVID-19, SARS-CoV-2.

\section{Introduction}

The spread of the SARS-CoV-2 infection has quickly and radically transformed health systems worldwide [1]. In recent years, telehealth's growth has been gradual, but the pandemic has led to an exponential increase, resulting in a rapid adoption of different telehealth strategies. The need to avoid crowding patients in health services and to offer alternative ways for patient assistance while preserving social distancing were compelling reasons for the growth of telehealth [2].

Brazil has the third highest number of COVID-19 cases worldwide, and the second highest number of deaths, with more than 8 million cases, and 200,000 deaths in January

\footnotetext{
${ }^{1}$ Corresponding Author: Antonio Luiz Pinho Ribeiro, Telehealth Center - Hospital das Clínicas - UFMG - Av. Professor Alfredo Balena, 110 - 1st Floor - South Wing - Room 107 30130-100 - Belo Horizonte - Minas Gerais, Brazil. Email: antonio.ribeiro@ebserh.gov.br(5531)33079201
} 
2021 [3]. In March 2020, the Brazilian Ministry of Health changed the telehealth regulations in response to the pandemic, allowing virtual interaction between healthcare professionals and patients in public and private healthcare, for pre-clinical care, assistance support, consultation, monitoring, and diagnosis [4].

The Telehealth Network of Minas Gerais (TNMG) is a large-scale Brazilian public telehealth service. It was implemented in 2005, providing support to primary care practitioners with teleconsulting and remote analysis of exams [5]. The TNMG currently attends 1,058 municipalities in nine Brazilian states, and has already analysed more than 5.5 million exams and 136,000 teleconsultations in various medical and non-medical specialties.

The TNMG acted quickly to assist professionals and the population in coping with COVID-19, mainly in low resource settings, developing integrated care systems focused on meeting the needs of local communities. Given that the isolation and monitoring of patients and contacts is a major challenge, due to the scarcity of confirmatory tests, community needs are mainly related to community education and management of mild and moderate suspected cases [7].

\section{Objective}

To describe the multimodal experiences and development of a public telehealth service during the COVID-19 pandemic.

\section{Methods}

\subsection{The TNMG Developments}

Several educational and assistance tools have been developed and made available, to be used in an integrated manner, by both health professionals and the general public. They are described in Figure 1, and comprise the following tools:

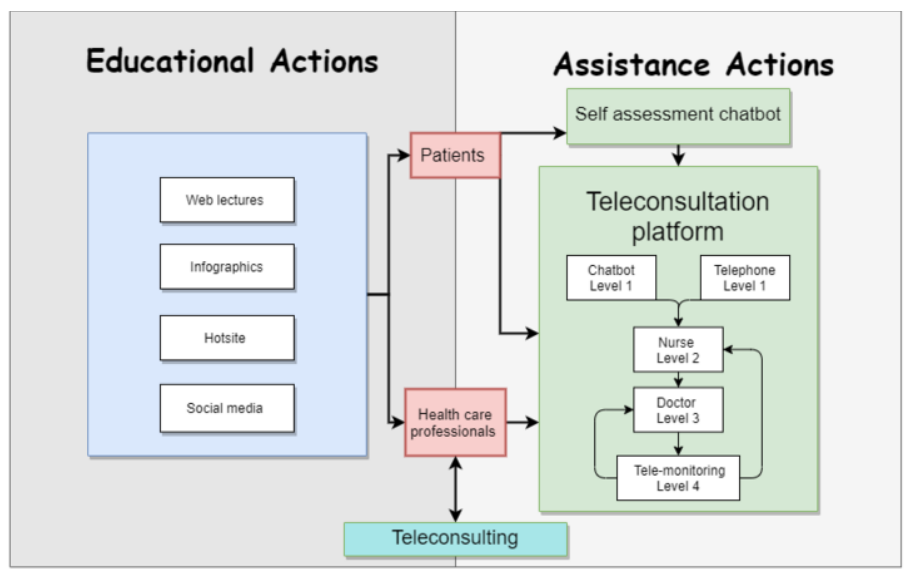

Figure 1. Integrated telehealth actions 
- A chatbot and a special telephone switchboard - a multifunction tool developed to clarify doubts about COVID-19, to perform a self-assessment of health status and a gateway to the teleconsultation/telemonitoring program, level 1 of care;

- A platform for teleconsultation combined with a telephone telemonitoring service, providing a comprehensive care with direct contact of the symptomatic patient with a multidisciplinary health team, and a follow-up of the patient and its contacts in home isolation, levels 2 (nursing), 3 (medical) and 4 (telemonitoring) of care;

- A teleconsulting service, for primary care practitioners to clarify doubts, related to specific COVID-19 patients or educational queries, with specialists;

- A tele-education program, including the development of infographics, educational videos and web lectures.

\subsection{The chatbot and the special telephone switchboard}

The clinical spectrum of COVID-19 is very broad, ranging from a simple cold to severe pneumonia, acute respiratory distress syndrome and death [8]. Based on data from the Chinese Center for Disease Control and Prevention, mild, severe and critical disease was reported in $81 \%, 14 \%$ and $5 \%$ of the cases in China [9]. Although asymptomatic infection has been reported, the exact proportion of these cases has not yet been defined. A recent systematic review has shown a prevalence of $20 \%$ and $75 \%$ in two general population studies, $8 \%$ to $50 \%$ in three studies with contacts, and $54 \%$ among nursing home residents [10]. No study so far has assessed the prevalence of asymptomatic COVID-19 infections in Brazil.

Screening of suspected cases and education of the population is necessary to avoid overload of health services with mild cases, and thus reduce the infection risk. A chatbot was developed to support the screening of suspected COVID-19 cases, as well as to promote education of the Brazilian population on the topic. The development team, composed of clinicians and IT personnel, focused on two key user experience design principles: (i) make it fast and easy for the user, with low technical barriers, without login or app installation, and request minimal user information; (ii) avoid medical jargon, to make it accessible for Brazilians with different levels of education and health literacy [11]. For the screening of suspected COVID-19, a decision algorithm was built by a team of clinicians of the TNMG, based on patients' symptoms, comorbidities, and national and international recommendations $[12,13]$.

The chatbot was developed in BLiP, and is available both through WhatsApp and at the TNMG's website [14]. Two different versions were developed: a stand-alone version, to directly advise and educate the user about general measures and symptom interpretation. As well as a teleassistance version; a digital front door to the teleconsultation and telemonitoring programs.

In addition to the chatbot, in selected cities where the teleconsultation program was being implemented, a special telephone switchboard was developed. The switchboard aimed to cater for the illiterate, those without smartphones, or individuals without the skills to use a chatbot. This telephone switchboard had the same functionality as the chatbot and played an educational role in clarifying doubts about COVID-19, guiding the population on the epidemiological situation, and functioning of municipal health services. Moreover, it also served as a gateway to the teleconsultation service. 


\subsection{The platform for teleconsultation and telemonitoring of symptomatic individuals}

The TNMG developed a specially designed platform for health status assessment and follow-up of individuals with respiratory symptoms. As shown in Figure 1, in addition to the chatbot and telephone front door (level 1), the platform has three more levels of care; level 2; nurse appointments; level 3, medical appointments; and level 4; telephone telemonitoring. An application was developed for use in a web environment. The teleconsultations were carried out by constantly trained doctors and nurses. The software allowed queries by video or telephone, which included the complete evaluation of signs and symptoms presented by the patient, including alarm signs, comorbidities, current medications, personal medical history, allergies, and the screening of household contacts. It also provided the option to make video recordings, prescriptions, medical certificates and reports, for the patient and contacts, as well as to request diagnostic tests for COVID19 , to schedule new teleconsultations, or to refer patients to telephone telemonitoring. Case notification forms and referral forms for health units and emergency services were also available. All the files were generated in a PDF format, so patients could easily download, print and use them, if necessary.

On completion of the medical teleconsultation the patient, if symptomatic and inhome isolation, was referred to telemonitoring. The telemonitoring service was carried out by properly trained medical students from local universities (i.e. telephone calls for patient follow-up), under the supervision of a qualified health professional. They received ongoing training by the program coordinator and made telephone contact with the user every 24 hours where patients presented decompensated comorbidities, pregnancy and puerperium, previous alarm signs or were aged over 60 years. Contact was made every 48 hours in the remaining cases. This service used the same technology developed for the teleconsultation service.

\subsection{The teleconsulting service}

TNMG has performed offline teleconsultations to support primary care practitioners since 2007 . Registered primary care practitioners of 4,931 primary care centers in remote municipalities access the TNMG website [14] via internet and present cases, and, whenever necessary, attach images or other files. Professionals of 46 medical and nonmedical specialties on duty in the TNMG receive the request, analyze it, and respond within 24 hours, or forward the request to a subspecialist, who responds within 48 hours [5]. As an action to support health professionals during the pandemic, a specific category was created for doubts about SARS-COV-2 and COVID-19.

\subsection{The tele-education program}

The tele-education team worked on various materials to support frontline workers and the general community. Infographics were designed and short educational videos were recorded, directed both to the population and to healthcare professionals. There were also live web lectures about COVID-19, taught by a reference professor in the field. Frequently Asked Questions (FAQs) were developed for healthcare professionals and the general population. All the educational materials produced were made available on a specific COVID-19 hot site on the TNMG platform [14], and all the educational videos were uploaded on TNMG's YouTube channel. Three times a week, short messages related to the topics were posted on Twitter, Facebook and Instagram. 


\section{Results}

The project had to be conducted in an extremely tight timescale and the service and educational program was made available for use in just two months after the start of development. All resources were made freely available on TNMG's website [14] and social media pages.

\subsection{The chatbot and the special telephone switchboard}

After the greeting message, the chatbot asked for the user's name, to make a semipersonalized friendly interaction, age, gender, location and contact phone number. Then, the user was asked whether the intention is an assessment of his/her symptoms or access to educational questions and answers about COVID-19. In the first case, the user terms are provided, including the information that there is no intention to substitute a medical consultation. If the user agreed, a sequence of questions was presented. To make the screening process simple and efficient, users were asked first whether they had warning symptoms that may be related to severe disease, then about conditions that may increase the risk of severe disease. According to what was selected in those steps, they were ranked in colors, which corresponded to the risk of complications.

According to the guidelines, dyspnea, hypotension and persistent fever were considered warning signs, and individuals presenting any of these should seek medical care $[12,13]$. So, these were the first questions presented to the user. In the selfassessment version of the chatbot, individuals who reported dyspnea or fainting sensation were ranked as 'red' and advised to seek emergency care as soon as possible. Individuals with persistent fever for more than three days or recrudescence of fever after 48 hours were ranked as 'orange' and advised to seek care in hospital settings.

The clinical guidelines recommend that individuals with comorbidities that increase the risk of severe disease should be assessed in reference centers [12,13]. Users without alarming signs but with upper airway symptoms were then asked about specific conditions, such as: cardiac disease (angina, previous myocardial infarction, heart failure), pulmonary disease (asthma, chronic pulmonary obstructive disease, cystic fibrosis), renal failure or dialysis, previous transplantation, cancer, rheumatic disease (lupus, rheumatoid arthritis or other), diabetes, HIV infection, Down syndrome, use of corticosteroids or immunosuppressants, pregnancy or postpartum and child aged under one-year-old. The individuals with any of these conditions were ranked as 'yellow', and in the self-assessment version of the chatbot advised to seek a reference center. Those without alarming signs and comorbidities were ranked as 'green' and received advice about how to deal with the mild disease, home isolation measures, need for rest and hydration. Except for the individuals ranked as 'red', all the other groups were offered the educational session from the chatbot.

The educational session of the chatbot was developed based on eighty-five frequently asked questions, from a database from the TNMG. The answers were developed by a group of medical doctors and professors, based on the best available evidence $[12,13,15]$. The questions were grouped into 12 topics: general information, virus transmission, COVID-19 symptoms, diagnosis, advice, treatment, house care, hygiene, lifestyle, mask use, pregnancy and pets. The database is constantly growing, and new topics, questions and answers are periodically added.

Currently, two medium-sized cities in the state of Minas Gerais have adopted the teleconsultation service provided by the TNMG, specially developed for the assistance 
of COVID-19 suspected cases. In these locations, a teleassistance version of the chatbot was implemented, connected to the teleconsultation service. The special telephone switchboard was also implemented in these two cities, as an alternative to the chatbot, providing the same educational and assistance services through trained operators. In this version, the chatbot, or the telephone number, is the front door to the teleconsultation program, called assistance level 1. The user classified as 'yellow' or 'green' is referred to a nurse appointment, the assistance level 2 . Whereas the user who fulfills the criteria for 'red' or 'orange' priority is directed to a teleconsultation with a medical doctor, the assistance level 3. Between May and November 2020, 1,212 calls via chatbot and 3,794 via telephone were made in these two cities. The chatbot algorithm is summarized in Figure 2.

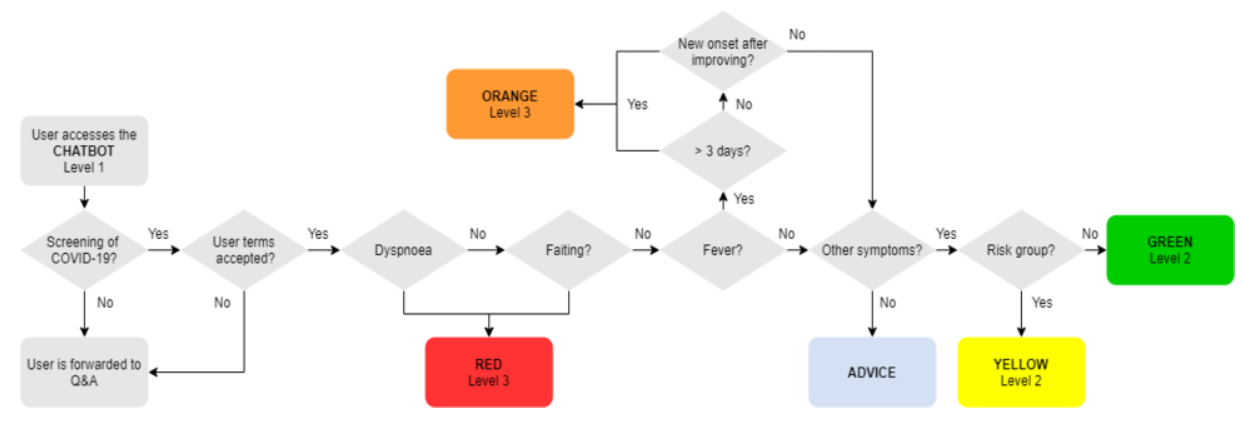

Figure 2. Chatbot decision algorithm

\subsection{The platform for teleconsultation and telemonitoring of symptomatic patients}

The service has been made available in two medium-sized cities in the state of Minas Gerais: Divinópolis and Teófilo Otoni. Divinopolis is located in the Midwest region of Minas Gerais, Brazil, with a population of 213,016 inhabitants and a human development index (HDI) of 0.76. The public health system is based on Primary Health Care (PHC) supported by Unified Heath System (SUS). This municipality is a health care referral center in a region comprising 54 municipalities. It has three private hospitals and two public, plus one hospital operating under an agreement with SUS and an emergency unit [16]. Teófilo Otoni is located in the Northeast of Minas Gerais, in the Mucuri Valley, with a population of 134,745 inhabitants, HDI of 0.70 . This municipality is also a health care referral center for specialized care in a region comprising 27 municipalities. It has three hospitals operating under an agreement with SUS and an emergency unit [17].

The teleconsultation service is being conducted with the support of well-trained doctors and nurses in all the appointments, in close partnership with local universities and municipal health offices in each city. The classification of severity levels is established through the color scale and creates, in specific software, a list of priorities for medical and nursing attendance. All of the consultations occur only after the patient has accepted the terms of consent.

The user classified as 'yellow' or 'green' is first referred to a nurse appointment, level 2 of assistance. The nursing team checks the symptoms and eventual comorbidities of interest through a detailed clinical interview, guiding self-home isolation with 
telemonitoring (level 4), doctor teleconsultation (level 3), or on-site evaluation in a health unit, in case they deem it necessary. They are also trained to clarify doubts, guide contact tracing and provide advice based on best practice guidelines $[12,13]$.

The user with alarming symptoms who meets the criteria for 'red' or 'orange' priority in the screening is directed to a teleconsultation with a medical doctor (level 3). The professional then checks whether there is an alarming sign and, in this case, refers the patient to an emergency unit. If there is no alarming sign, the doctor can choose to manage the patient through online care only, with other teleconsultations appointments and telemonitoring. Prescriptions, medical reports, and confirmatory testing requests, both for the patient and contacts, are provided.

From May to November 2020, 5,200 attendances of 4,371 patients were made at level 2, and 4,053 attendances of 2,994 patients at level 3. All patients seen in the teleconsultation program, for whom the self-home isolation with symptomatic treatment was recommended, were included in the telemonitoring service of the TNMG, for at least 10 days. In both cities, the telemonitoring service receives patients initially evaluated in the Conventional Health Centers, who were referred to the telehealth service with a direct entry at level 4 . Therefore, in the two cities where the program has been established, it has become the main tool for monitoring COVID-19 patients in the public health service.

Patients who were monitored by the telemonitoring team received guidance about the length of isolation, the required restrictions and about how to proceed within contact with other people in the household, in order to minimize the spread of the virus, as recommended by the Brazilian Ministry of Health [13]. They also received advice concerning mental and physical health, such as healthy eating and physical activity. All users are instructed to seek an on-site assessment in case of clinical deterioration or redirected to the teleconsultation program in case of any need.

During the monitoring period, 22,412 attendances were made, monitoring more than 7,000 symptomatic patients in both cities. Preliminary analyses revealed that this number represents more than $90 \%$ of the symptomatic patients who entered the system, showing a high effectiveness of the service in reducing the demand of these patients for an on-site evaluation.

\subsection{The teleconsulting service}

Primary care practitioners from 728 cities in Minas Gerais State could send their doubts about COVID-19 through TNMG's website [14]. The doubts could be about specific clinical cases or general doubts, and an infectious disease specialist answered the queries within 24 hours. If necessary, educational material was forwarded together with the teleconsulting response. From May to November 2020, guidance was provided to 979 queries specifically related to COVID-19.

\subsection{The tele-education program}

A description of the educational materials produced is shown in Table 1. The educational videos have reached on average more than 108,000 views on YouTube. Six live web lectures about COVID-19 were seen in real-time by 20,000 professionals, mainly nurses $(63.8 \%)$ and doctors $(31.1 \%)$, from 45 medical specialties (most family physicians$34.0 \%$ ), in all Brazilian states, especially the Southeast region (58.5\%). Each one of the lectures has reached 5,300 views on average. The hot site has received over 43,000 hits to date. 
Table 1. Production of Educational Material

\begin{tabular}{cc}
\hline Material & Amount \\
\hline Short educational videos & 21 \\
Infographics & 20 \\
Web lectures & 6 \\
FAQ & 150 \\
Clinical protocols & 2 \\
\hline
\end{tabular}

\section{Discussion}

The development and implementation of multiple integrated telehealth strategies during the COVID-19 pandemic has been a complex and much-needed task. As in other areas, from basic science to clinical trials, the pandemic has imposed a new pace of knowledge building in telehealth, leading to the creation of numerous innovative tools in record time. Such growth is only possible through highly coordinated multidisciplinary work, which involves IT professionals, professors, researchers, doctors, nurses, health managers, local health workers and students.

Several positive aspects of its implementation and operation can be highlighted. First, we must emphasize the alignment with health planning policies and the multidimensional character of the actions [14]. The association of direct health care actions with educational campaigns both for the population and primary health care professionals provides not only a tool that guarantees quality care to the population but also autonomy for users and professionals, with workforce development. The health team of teleconsultation has identified places with possible outbreak of COVID-19 and notified municipalities in a timely manner. Second, centralized coordination, in this interconnected network with multiple collaborators involved, maintains in the TNMG a coordinating center that manages and audits health care, educational and IT development actions, in close interface with municipal health centers. Third, the accessibility of the tools is a key point, both for the simplicity of the devices needed, an instant messaging app or a phone if limited access to the internet, and for the simple and accessible language used. Making it possible to reach a diverse population, of different educational levels. The use of low-cost systems with web platforms allowed for the development of lowcost services in a short time period. Lastly, longitudinal monitoring of patients included in the teleconsultation and telemonitoring system allowed home isolation of patients with the safety of clinical follow-up, necessary due to the sub-acute and prolonged nature of the disease [18]. These actions, contributed to expanding access to timely medical assessment, to the reduction of the proportion of patients in need of on-site evaluation, and to better organize health services. Therefore, it might have contributed to the reduction of virus circulation, keeping at-risk people safe.

The program implementation has also faced many challenges. Unfortunately, due to the urgency of the situation, it was not possible to conduct validation studies of the platforms or software prior to implementation, nor was it possible to involve patients in the development of the self-assessment tools [19]. It was considered an achievement to be able to utilize the platform following a short development period.

The caution to not interfere in local health care networks already implemented and established has been a point of contention. In order to avoid this situation, the local community of health workers and the local university community have been fully involved in the assistance actions. In addition, the alignment of the scientifically based protocols developed by the TNMG with local routines of COVID-19 care, which 
sometimes did not align, has been a topic of concern. As the information changes and knowledge base expands, there is the need to constantly update the system. Thus, continuous remote training of the team is required, both on the use of the systems and the development of assistance protocols. These challenges have been overcome, and the great integration between the telehealth center and the local health workers community is now the major aspect of the program's success.

Another point of attention has been the lack of resources, especially in one of the municipalities served by the program, Teófilo Otoni. The poor telephone network and the lack of access to internet $4 \mathrm{G}$ technology in the city has led to unsuccessful calls and loss of follow-up. Also because of inherent characteristics of the underserved communities, more complex and elaborate platforms that allow, for example, scheduling appointments directly in the system by the patient himself, were not feasible. The platforms developed are particularly appropriate to the general management of COVID19 in primary care, and not very suitable for handling complex populations in need of highly specialized care. However, this can be a first step towards the incorporation of the technology in other health care settings, as a regular and permanent service.

To date, at the beginning of 2021, Brazil faces an important increase in the number of COVID-19 cases, the services remain in full operation, reaching their peak of activity. Therefore, an objective analysis of the final impact of their implementation is not yet possible and is planned for the upcoming months. In parallel, the user satisfaction will be objectively analyzed, using information collected during appointments.

\section{Conclusion}

The COVID-19 pandemic is having a profound impact on health systems around the globe. Challenges are imposed both by the burden that severely ill patients bring to the hospital systems and by the need for social distance and consequent remote management of less severe cases. Also, the dissemination of reliable and updated information to the population is a tough and essential task. In the COVID-19 era, telehealth tools have become great alternatives to provide appropriate care to patients. The TNMG services appear to be efficient and robust during the health crisis of COVID-19, through different tools and methodologies focused on both professionals and users of the health systems, with teleguidance, teleconsultations, telemonitoring, teleconsulting and tele-education. Telehealth services should continue to be part of medium and long-term care and become a permanent part of the available supportive resources.

\section{Funding sources}

This project was funded by Legislative Chamber of the State Government of Minas Gerais (Assembléia Legislativa de Minas Gerais), by CAPES (Coordenação de Aperfeiçoamento de Pessoal de Nível Superior, process 88887.507149/2020-00) and FAPEMIG (Fundação de Amparo à Pesquisa de Minas Gerais, RED-00081-16). Dr. Ribeiro was supported in part by National Council for Scientific and Technological Development (Conselho Nacional de Desenvolvimento Científico e Tecnológico, CNPq, 310679/2016-8 and 465518/2014-1), by FAPEMIG (PPM-00428-17). 


\section{References}

[1] Emanuel EJ, Persad G, Upshur R, Thome B, Parker M, Glickman A, et al. Fair Allocation of Scarce Medical Resourcesin the Time of Covid-19. NEnglJMed. 2020 March; 382(21):2049-55.

[2] Hollander JE, Carr BG. Virtually Perfect? Telemedicine for Covid-19. N Engl J Med. 2020 April; 383(18):1679-81.

[3] Ministério da Saúde - Coronavírus Brasil. Painel de casos de doença pelo coronavírus 2019 (COVID-19) no Brasil pelo Ministério da Saúde [Accessed Jan 12, 2021]. Available: https:/covid.saude.gov.br/.

[4] Ministério da Saúde (Brasil). Portaria $n^{\circ}$ 467, march 20, 2020. Dispõe, em caráter excepcional e temporário, sobre as ações de Telemedicina, com o objetivo de regulamentar e operacionalizar as medidas de enfrentamento da emergência de saúde pública de importância internacional. DiárioOficial da União March 23 2020; Seção 1.

[5] Alkmin MB, Figueira RM, Marcolino MS, Cardoso CS, Abreu MP, Cunha LR, et al. Improving patient access to specialized health care: the Telehealth Network of Minas Gerais, Brazil. Bull World Health Organ. 2012 May 1;90(5):373-8.

[6] Guimarães VHA, Leandro MO, Cassiano C, Marques ALP, Motta C, Freitas-Silva AL, et al. Population's Knowledge About COVID-19: A Cross-Sectional Web-Based Study in Brazil. JMIR Public Health Surveill. 2020 Dec; doi:10.2196/24756.

[7] Thornton, J. Covid-19: Lack of testing in Brazil is a "major failure," says MSF. BMJ 2020;370:m2659.

[8] Borges do Nascimento IJ, von Groote TC, O’Mathúna DP, Abdulazeem HM, Henderson C, Jayarajah U, Weerasekara I, et al. Clinical, laboratory and radiological characteristics and outcomes of novel coronavirus (SARS-CoV-2) infection in humans: A systematic review and series of meta-analyses. PLoS ONE. 2020 Sept. 15(9): e0239235.

[9] $\mathrm{Wu} \mathrm{Z}$, McGoogan JM. Characteristics of and Important Lessons From the Coronavirus Disease 2019 (COVID-19) Outbreak in China: Summary of a Report of 72314 Cases From the Chinese Center for Disease Control and Prevention. JAMA. 2020;323(13):1239-1242.

[10] Yanes-Lane M, Winters N, Frogonese F, Bastos M, Perlman-Arrow S, Campbell JR, Menzies D. Proportion of asymptomatic infection among COVID-19 positive persons and their transmission potential: A systematic review and meta-analysis. PLoS One. 2020; Nov 3;15(11):e0241536.

[11] Kocaballi AB, Berkovsky S, Quiroz JC, Laranjo L, Tong HL, Rezazadegan D, et al. The Personalization of Conversational Agents in Health Care: Systematic Review. J Med Internet Res. 2019;21(11):e15360.

[12] World Health Organization. Clinical Management of Covid-19: interim guidance. Geneva: WHO, 2020.

[13] Ministério da Saúde. Orientações para manejo de pacientes com Covid-19. Brasília, 2020.

[14] Centro de Telessaúde do Hospital das Clínicas da Universidade Federal de Minas Gerais [Web page] [accessed in jan 07, 2020]. Available in: https://telessaude.hc.ufmg.br/.

[15] UpToDate [Web page]. Coronavirus disease 2019 (COVID-19) - Home [accessed in jan 07, 2021]. Available in: https://www.uptodate.com/landing/covid19?source=promobox.

[16] Costa MM, Pádua CAM, Figueiredo RC, Rocha BG, Faria ML, et al. Drug Use Profile and Mortality in Patients with Diabetes Mellitus. Journal of Pharmacy and Pharmacology. 2020; 8, 99-109.

[17] Ricardo HB. Educação em saúde com pacientes hipertensos, na Equipe de Saúde da Família Topazio, Teófilo Otoni, Minas Gerais. TCC (Curso de Especialização em Gestão do Cuidado em Saúde da Família). Universidade Federal de Minas Gerais, Teófilo Otoni. 2018

[18] Cohen P, Blau J. Coronavirus disease 2019 (COVID-19): Outpatient evaluation and management in adults. In: UpToDate, Post TW (Ed), UpToDate, Waltham, MA. (Accessed on January 10, 2021.)

[19] Gray CS, Tang T, Armas A, Backo-Shannon M, Harvey S, Kuluski K, et al. Building a Digital Bridge to Support Patient-Centered Care Transitions From Hospital to Home for Older Adults With Complex Care Needs: Protocol for a Co-Design, Implementation, and Evaluation Study. JMIR Res Protoc. 2020 Nov; 9(11):e20220. 